\title{
TRANSFINITE METHODS IN METRIC FIXED-POINT THEORY
}

\author{
W. A. KIRK \\ Received 28 September 2001
}

This is a brief survey of the use of transfinite induction in metric fixed-point theory. Among the results discussed in some detail is the author's 1989 result on directionally nonexpansive mappings (which is somewhat sharpened), a result of Kulesza and Lim giving conditions when countable compactness implies compactness, a recent inwardness result for contractions due to Lim, and a recent extension of Caristi's theorem due to Saliga and the author. In each instance, transfinite methods seem necessary.

\section{Introduction}

George Cantor introduced the process of transfinite induction over one hundred years ago ([4], see also [8]) despite the fact that at that time the question of whether any set could actually be well ordered remained unresolved. Since then, this powerful technique has found many applications in analysis and topology. In this paper, we review some of its more recent applications in metric fixedpoint theory. The results obtained in this way may not always be the most interesting, but they tend to be "sharp."

Some authors prefer transfinite induction as a standard mode of argument. Usually, however, the process is invoked when other methods either fail or are not available, or when some iterative process almost invites the approach. Many results initially obtained via transfinite induction arguments, such as Caristi's theorem [5] and Lim's theorem for multivalued nonexpansive [23], have later been derived by more elegant methods, and in some instances under weaker logical assumptions (e.g., see [12]). In this survey, we focus on four recent examples for which there seems to be no alternative approach. These are, in chronological order, the transfinite extension of Ishikawa's iteration scheme given in [15], the Kulesza-Lim countable weak compactness result of [21], Lim's weak inwardness 
312 Transfinite methods in metric fixed-point theory

result of [23], and a recent extension of Caristi's theorem given in [20]. The paper concludes with some historical comments.

This paper is largely expository although Theorem 2.4 is a new formulation, and in this case we give a detailed proof.

\section{2. "Directional" contractions}

We begin by looking back at the transfinite method of our 1989 MarseilleLuminy paper [15]. That approach arose in an attempt to sharpen results about "weak directional contractions."

For points $x, y$ of a metric space $(M, d)$, we denote

$$
(x, y)=\{z \in M: d(x, z)+d(z, y)=d(x, y) \text { and } x \neq z \neq y\} .
$$

The metric space $M$ is said to be metrically convex if $(x, y) \neq \varnothing$ whenever $x, y \in M, x \neq y$.

The mapping $T: M \rightarrow M$ is said to be pointwise Lipschitzian on $M$ with constant $k$ if $T$ is continuous, and for each $x \in M$,

$$
\lim \sup _{\substack{y \rightarrow x \\ y \neq x}} \frac{d(T(x), T(y))}{d(x, y)} \leq k,
$$

$T$ is called a pointwise contraction if $k \in[0,1)$.

The mapping $T: M \rightarrow M$ is said to be almost directionally Lipschitzian on $M$ with constant $k$ if for each $x, y \in M$,

$$
\inf _{z \in(x, y)} \frac{d(T(x), T(z))}{d(x, z)} \leq k .
$$

The mapping $T: M \rightarrow M$ is said to be weakly directionally Lipschitzian on $M$ with constant $k$ if $T$ is continuous, and for each $x \in M$,

$$
\lim _{z \rightarrow x} \inf _{z \in(x, T(x))} \frac{d(T(x), T(z))}{d(x, z)} \leq k
$$

$T$ is a weak directional contraction if $k<1$. In [6], Clarke proved that every weak directional contraction, defined on a complete metric space, has a fixed point. He asked whether pointwise contractions on a complete and convex metric spaces are global contractions. The following answers this in the affirmative. (An example of a weak directional contraction that is not a contraction is given in [6].) 
Theorem 2.1 (Kirk-Ray [19]). Let $M$ and $N$ be complete metric spaces with $M$ metrically convex, and suppose $T: M \rightarrow N$ is almost directionally Lipschitzian with constant $k$. Suppose, in addition, that $T$ is a closed mapping (i.e., has a closed graph). Then $T$ is Lipschitzian with global Lipschitz constant $k$.

A Banach space version of the above result was given in an earlier paper [18]. In both instances, transfinite induction arguments were used although it is possible to give proofs of these results based on Zorn's lemma.

The above considerations motivated the approach of [15]. Now we assume that $K$ is a bounded closed convex subset of a Banach space, and here we use $S(x, y)$ to denote the set $\{(1-\alpha) x+\alpha y: \alpha \in(0,1)\}$.

Definition 2.2. A mapping $T: K \rightarrow K$ is said to be weakly directionally nonexpansive on $K$ if $T$ is continuous, and for each $x \in M$,

$$
\|T(x)-T(z)\| \leq\|x-z\|
$$

for all $z \in S(x, T(x))$ sufficiently near $x$.

Theorem 2.3 (Kirk [15]). Let $K$ be a bounded closed and convex subset of a $\mathrm{Ba}$ nach space and suppose $T: K \rightarrow K$ is weakly directionally nonexpansive on $K$. Then $\inf \{\|x-T(x)\|: x \in K\}=0$.

Now we show how the method of [15] can be used to prove a minor variant of the above result. The proof serves to illustrate the delicate nature of the transfinite argument. Here we assume that the mapping is locally nonexpansive. By this we mean that each point has a neighborhood such that the restriction of the mapping to that neighborhood is nonexpansive. The domain is not assumed to be convex otherwise the mapping would be globally nonexpansive. The condition on $T$ is an "inwardness" type assumption-see Section 4.

Theorem 2.4. Let $D$ be a bounded closed subset of a Banach space $X$ and let $T$ : $D \rightarrow X$ be a locally nonexpansive mapping. Suppose also that

$$
x \in D \Longrightarrow x-h(x-T(x)) \in D \quad \forall h>0 \text { sufficiently small. }
$$

Then $\inf \{\|x-T(x)\|: x \in D\}=0$.

We will apply the following lemma, which is Lemma 2.1 of [15]. This is a transfinite version of a 1976 result basically due to Ishikawa [13].

Lemma 2.5. Let $X$ be a Banach space, $D \subset X, \Omega_{1}$ the set of countable ordinals, and $\gamma \in \Omega_{1}$. For each $\alpha<\gamma$, suppose $\left\{x_{\alpha}\right\}$ and $\left\{y_{\alpha}\right\}$ in D satisfy

(i) $x_{\alpha+1}=\left(1-t_{\alpha}\right) x_{\alpha}+t_{\alpha} y_{\alpha}$ for some $t_{\alpha} \in(0,1)$;

(ii) $\left\|y_{\alpha}-y_{\alpha+1}\right\| \leq\left\|x_{\alpha}-x_{\alpha+1}\right\|$; 
314 Transfinite methods in metric fixed-point theory

(iii) if $\mu<\gamma$ is a limit ordinal, then

$$
\lim _{\alpha>\mu} x_{\alpha}=x_{\mu}, \quad \lim _{\alpha>\mu} y_{\alpha}=y_{\mu}
$$

Suppose further that for each $\mu<\gamma, \sum_{\alpha<\mu} t_{\alpha}<\infty$.

Then

$$
\begin{aligned}
& \left(1+\sum_{\alpha \leq s \leq \alpha+\beta} t_{s}\right)\left\|y_{\alpha}-x_{\alpha}\right\| \\
& \quad \leq\left\|y_{\alpha+\beta+1}-x_{\alpha}\right\|+\prod_{\alpha \leq s \leq \alpha+\beta}\left(1-t_{s}\right)^{-1}\left[\left\|y_{\alpha}-x_{\alpha}\right\|-\left\|y_{\alpha+\beta+1}-x_{\alpha+\beta+1}\right\|\right] .
\end{aligned}
$$

Moreover, if $D$ is bounded and $t_{\alpha} \leq b<1$ for each $\alpha<\gamma$, then the assumption $\sum_{s<\gamma} t_{s}=+\infty$ implies $\lim _{\alpha>\gamma}\left\|y_{\alpha}-x_{\alpha}\right\|=0$.

Proof of Theorem 2.4. By assumption, each point $x \in D$ has a neighborhood $N(x)$ such that the restriction of $T$ to $N(x) \cap D$ is nonexpansive. Let $\varepsilon>0$. We assume $\|x-T(x)\|>\varepsilon$ for each $x \in D$ and show that this leads to a contradiction. Let $\Omega_{1}$ denote the smallest uncountable ordinal and fix $b \in(0,1)$. Suppose $\left\{x_{\mu}\right\},\left\{y_{\mu}\right\}$ have been defined in $K$ for all $\mu<\gamma \in \Omega_{1}$ so that (i), (ii), and (iii) of Lemma 2.5 hold, where $\left\{t_{\mu}\right\} \subset(0, b)$, and $y_{\mu}=T\left(x_{\mu}\right)$. By assumption, $\left\|x_{\mu}-y_{\mu}\right\|>\varepsilon$. If $\gamma=$ $\beta+1$ for some $\beta$, apply condition (2.8) of Lemma 2.5 to choose $t_{\beta} \in(0, b)$ so that if

$$
z=(1-t) x_{\beta}+t T\left(x_{\beta}\right)
$$

for $0<t \leq t_{\beta}$, then $z \in N\left(x_{\beta}\right) \cap D$ and set

$$
x_{\beta+1}=\left(1-t_{\beta}\right) x_{\beta}+t_{\beta} T\left(x_{\beta}\right) \text {. }
$$

Then $x_{\beta+1} \in D$. Now set $y_{\beta+1}=T\left(x_{\beta}\right)$. Now suppose $\gamma$ is a limit ordinal. Lemma 2.5 excludes the possibility $\sum_{0 \leq s<\gamma} t_{s}=+\infty$ since $\left\|x_{\mu}-y_{\mu}\right\|>\varepsilon$. On the other hand, if $\sum_{0 \leq s<\gamma} t_{s}<+\infty$, then $\left\{x_{\mu}\right\}_{\mu<\gamma}$ is a Cauchy net (this is proved in [15]) so, it is possible to define $x_{\gamma}=\lim _{\mu r \gamma} x_{\mu}$ and, since $T$ is continuous,

$$
y_{\gamma}=\lim _{\mu^{\prime} \gamma} y_{\mu}=\lim _{\mu^{\prime} \gamma} T\left(x_{\mu}\right)
$$

Now we have $\left\{x_{\mu}\right\}$ and $\left\{y_{\mu}\right\}$ defined for all $\mu \in \Omega_{1}$. Next observe that

$$
\begin{aligned}
\left\|y_{\alpha+1}-x_{\alpha+1}\right\| & \leq\left\|y_{\alpha+1}-y_{\alpha}\right\|+\left\|y_{\alpha}-x_{\alpha+1}\right\| \\
& \leq\left\|x_{\alpha+1}-x_{\alpha}\right\|+\left\|y_{\alpha}-x_{\alpha+1}\right\| \\
& =\left\|y_{\alpha}-x_{\alpha}\right\| .
\end{aligned}
$$


Thus, $r:=\lim _{\alpha}\left\|y_{\alpha}-x_{\alpha}\right\|$ exists. However, the set

$$
\left\{\alpha \in \Omega_{1}:\left\|y_{\alpha+1}-x_{\alpha+1}\right\|<\left\|y_{\alpha}-x_{\alpha}\right\|\right\}
$$

can be at most countable. Therefore, there exists $\gamma \in \Omega_{1}$ such that for $\alpha \geq \gamma$, $\left\|y_{\alpha}-x_{\alpha}\right\| \equiv r$, and by assumption, $r \geq \varepsilon>0$. On the other hand, condition (2.8) of Lemma 2.5 now implies

$$
\left(1+\sum_{\gamma \leq \alpha<\Omega_{1}} t_{\alpha}\right) r \leq \operatorname{diam}(D) .
$$

Since $t_{\alpha}>0$ for each $\alpha$ and $\Omega_{1}$ is uncountable, this is a contradiction.

\section{The Kulesza-Lim theorem}

Now we describe the result of Kulesza and Lim [21], a result is motivated by the following question. Are there normal structure type conditions, weaker than hyperconvexity, yet strong enough to assure that the intersection of any descending chain of nonempty admissible sets in a metric space is nonempty (and admissible)? The Kulesza-Lim result shows that if the underlying metric space is complete, the answer is yes. This is an instance where the very nature of the problem calls for transfinite methods.

We need some definitions and notation. Let $(M, d)$ be a bounded metric space and

$$
B(x ; r)=\{u \in M: d(u, x) \leq r\} .
$$

We use $\mathscr{A}(M)$ to denote the family of all admissible subsets of $M$. Thus,

$$
D \in \mathscr{A}(M) \Longleftrightarrow D=\bigcap_{i \in I} B\left(x_{i} ; r_{i}\right),
$$

where $x_{i} \in M, r_{i}>0$, and $i \in I$ (some index set). For $D \in \mathscr{A}(M)$, define

$$
r(D)=\inf \{r>0: D \subseteq B(x ; r) \text { for some } x \in D\} .
$$

Definition 3.1. The family $\mathscr{A}(M)$ is said to have normal structure if $r(D)<$ $\operatorname{diam}(D)$ whenever $\operatorname{diam}(D)>0 ; A(M)$ is said to have uniform normal structure if there exists $c \in(0,1)$ such that $r(D) \leq c \operatorname{diam}(D)$ for each $D \in \mathscr{A}(M)$.

Definition 3.2. The family $\mathscr{A}(M)$ is said to be countably compact (resp., compact) if every descending sequence (resp., chain) of nonempty sets in $A(M)$ has nonempty intersection.

Theorem 3.3. Suppose $(M, d)$ is a bounded complete metric space for which $\mathscr{A}(M)$ is uniformly normal. Then $\mathscr{A}(M)$ is compact. 
The starting point is the following, which can be proved using elementary methods.

Lemma 3.4 (Khamsi [14]). If $\mathscr{A}(M)$ is uniformly normal, then $\mathscr{A}(M)$ is countably compact.

Passing from countable compactness to compactness requires an escalation of the method of argument. The key idea in the proof of Theorem 3.3 is the following fact. Any uncountable chain of real numbers which is either strictly decreasing or strictly increasing is eventually constant. The following routine observation is needed for the proof.

LeMmA 3.5. If $\mathscr{A}(M)$ is countably compact but not compact, then there exists an uncountable ordinal $\Gamma$ and a descending transfinite chain $\left\{D_{\alpha}, \alpha<\Gamma\right\}$ of nonempty members of $\mathscr{A}(M)$ such that

$$
\bigcap_{\alpha<\Gamma} D_{\alpha}=\varnothing
$$

The set of all ordinals for which the conclusion of Lemma 3.5 holds is nonempty, and thus by well-ordering, there is a smallest such ordinal which we again call $\Gamma$. A chain $\left\{D_{\alpha}\right\}$ that satisfies the conclusions of Lemma 3.5 for $\Gamma$ is called a Г-chain.

Given a $\Gamma$-chain $\left\{D_{\alpha}\right\}$, for each $\alpha \in \Gamma$, let $d_{\alpha}=\operatorname{diam}\left(D_{\alpha}\right)$. Since $\left\{d_{\alpha}\right\}_{\alpha<\Gamma}$ is nonincreasing, $\lim _{\alpha} d_{\alpha}=d$ (in fact for $\alpha$ sufficiently large, $d_{\alpha} \equiv d$ ). Call $d$ the diameter of $\left\{D_{\alpha}\right\}$ and write

$$
d=\operatorname{diam}\left(\left\{D_{\alpha}\right\}\right)
$$

Finally, we say that a $\Gamma$-chain $\left\{J_{\alpha}\right\}$ is a refinement of a $\Gamma$-chain $\left\{D_{\alpha}\right\}$ if $J_{\alpha} \subseteq D_{\alpha}$ for each $\alpha<\Gamma$. This leads to the following lemma.

LeMma 3.6. Under the hypothesis of Lemma 3.5, there exists a $\Gamma$-chain $\left\{D_{\alpha}\right\}$ with the property: if $\left\{J_{\alpha}\right\}$ is a refinement of $\left\{D_{\alpha}\right\}$, then $\operatorname{diam}\left(\left\{J_{\alpha}\right\}\right)=\operatorname{diam}\left(\left\{D_{\alpha}\right\}\right)$.

Lemmas 3.5 and 3.6 are then used to prove the following result which, in conjunction with Lemma 3.4, gives Theorem 3.3.

Theorem 3.7 ([21]). Suppose $(M, d)$ is a bounded metric space for which $\mathscr{A}(M)$ is countably compact and normal. Then $\mathscr{A}(M)$ is compact.

We mention that a result similar in spirit to the above is found in [16]. Transfinite methods are used there as well. The result of [16] is formulated for topologies defined by a collection of sets which may properly contain the admissible sets. A convexity structure $\Sigma$ on a metric space $M$ is a family of subsets of $M$ that contains $M$, contains the closed balls of $M$, and is closed under intersections. A proximinal set in $\Sigma$ is a set (in $\Sigma$ ) which lies on the boundary of some closed ball in $D$. 
Theorem 3.8 ([16]). Let $(M, d)$ be a bounded metric space, let $\Sigma$ be a countably compact convexity structure which contains its closed $r$-neighbors, and let $\tau$ be the topology on $M$ generated by $\Sigma$ as a subbase for closed sets. Then if the proximinal sets in $\Sigma$ are either separable or quasinormal, $M$ is $\tau$-compact.

\section{Lim's inwardness result}

Now we turn to Lim's recent inwardness result. This too is a theorem for which the standard mode of proof seems to fail. It may be too much to say that it requires a transfinite induction argument, but it does seem clear that a straightforward application of Caristi's theorem is not adequate. We will illustrate this in detail.

Theorem 4.1. Let $D$ be a nonempty closed subset of a Banach space $X$ and let $T: D \rightarrow 2^{X} \backslash\{\varnothing\}$ be a multivalued contraction with closed values which is weakly inward on $D$. Then $T$ has a fixed point.

The above result was proved by Martínez-Yañez in 1991 [24] for single-valued $T$, by Yi and Zhao in 1994 [35] for compact-valued T, and by Xu in 2001 [34] for $T$ satisfying the condition that each set $T x$ is proximinal relative to $x$. In each of these instances, it was possible to apply Caristi's theorem directly. Reich [29] also uses Caristi's theorem to give an extension of Lim's result in [22] to certain inward maps. (See [33] for another exposition on the ideas of this section.)

For a closer look at this result, we define the terms. Let $T$ be a nonempty closed subset of a Banach space $X$ and $T: D \rightarrow 2^{X} \backslash\{\varnothing\}$, a multivalued contraction mapping with closed values. Thus, there exists $k \in(0,1)$ such that

$$
H(T x, T y) \leq k\|x-y\|
$$

for all $x, y \in D$, where $H$ denotes the (extended) Hausdorff metric on the nonempty closed subsets of $X$. Thus,

$$
H(A, B)=\max \left\{\rho>0: A \subseteq N_{\rho}(B) \text { and } B \subseteq N_{\rho}(A)\right\},
$$

where

$$
N_{\rho}(S)=\left\{u \in X: \inf _{x \in S}\|u-x\| \leq \rho\right\} .
$$

The condition on $T$ assures that if some value of $T$ is bounded, then all are. It is well known that in this case, $T$ takes values in the complete metric space of all bounded nonempty closed subsets of $X$ endowed with the Hausdorff metric. On the other hand, if $T x$ is unbounded for some $x \in D$, then the space of all nonempty closed subsets of $X$, having finite Hausdorff distance from $T x$, is also a complete metric space.

The inward set of $D$ relative to $x \in D$ is the set

$$
x+\{\lambda(z-x): z \in D, \lambda \geq 1\} .
$$


318 Transfinite methods in metric fixed-point theory

$T$ is said to be weakly inward on $D$ if for each $x \in D$,

$$
T x \subseteq x+\overline{\{\lambda(z-x): z \in D, \lambda \geq 1\}} .
$$

Note that

$$
w=x+\lambda(z-x) \Longleftrightarrow w=\lambda z+(1-\lambda) x \Longleftrightarrow z=\frac{1}{\lambda} w+\left(1-\frac{1}{\lambda}\right) x .
$$

Therefore, the inward set of $D$ relative to $x$ consists of $D$ along with those points $w \in X \backslash D$ which have the property that some point $z \in D$ with $z \neq x$ lies on the segment joining $x$ and $w$.

$T$ is said to be weakly inward on $D$ if for each $x \in D, T$ lies in the closure of the inward set of $D$ relative to $x$, that is,

$$
T x \subseteq x+\overline{\{\lambda(z-x): z \in D, \lambda \geq 1\}} .
$$

Note that in Deimling [9] and elsewhere, $T: D \rightarrow 2^{X} \backslash\{\varnothing\}$ is called weakly inward if for each $x \in D$,

$$
T x \subseteq x+S_{D}(x)
$$

where

$$
S_{D}(x)=\left\{y \in X: \lim _{\lambda \rightarrow 0^{+}} \inf \lambda^{-1} \operatorname{dist}(x+\lambda y, D)=0\right\} .
$$

Lim has observed that it is always the case that

$$
S_{D}(x) \subseteq \overline{\{\lambda(z-x): z \in D, \lambda \geq 1\}}
$$

In fact, for convex $D$, the two concepts coincide (see $[5,28]$ ) but this is not true in general (see [9, Example 11.1]).

First we approach the proof of Theorem 4.1 with a view of applying Caristi's theorem which we now state.

Theorem 4.2 (Caristi). Let $(M, d)$ be a complete metric space and suppose $g: M \rightarrow$ $M$ is an arbitrary mapping which satisfies

$$
d(x, g(x)) \leq \varphi(x)-\varphi(g(x))
$$

for all $x \in M$ where $\varphi: M \rightarrow R$ is a lower semicontinuous mapping which is bounded below. Then $g$ has a fixed point in $M$.

Now we assume that $T$ satisfies the assumptions of Theorem 4.1. Let $\ell \in(k, 1)$ where $k$ is the Lipschitz constant of $T$ and choose $\varepsilon \in(0,1)$ so that

$$
b:=\frac{1-\varepsilon}{1+\varepsilon}-\ell>0
$$


Let $x \in D$. In order to apply Caristi's theorem, we would like to show that if $T$ is fixed point free, then it is possible to choose $g(x) \in D$ so that

$$
b\|x-g(x)\| \leq \operatorname{dist}(x, T x)-\operatorname{dist}(g(x), T \circ g(x)) .
$$

Toward this end, let $\varepsilon^{\prime}>0$ and choose $y \in T x$ so that

$$
\|x-y\| \leq \operatorname{dist}(x, T x)+\varepsilon^{\prime} .
$$

Since $T$ is fixed point free, $\operatorname{dist}(x, T x)>0$, so by the weak inwardness of $T$, there exist $g(x) \in D$ and $\lambda \geq 1$ such that

$$
\|x+\lambda(g(x)-x)-y\| \leq \varepsilon \operatorname{dist}(x, T x) .
$$

Since $\varepsilon \in(0,1)$, this in particular implies $g(x) \neq x$. Then if $\mu=\lambda^{-1}$,

$$
\|x-g(x)\|-\mu\|x-y\| \leq \varepsilon \mu \operatorname{dist}(x, T x)
$$

from which $\|x-g(x)\| \leq(1+\varepsilon) \mu \operatorname{dist}(x, T x)+\mu \varepsilon^{\prime}$.

Moreover, if $z=\mu y+(1-\mu) x$, then $\|g(x)-z\| \leq \varepsilon \mu \operatorname{dist}(x, T x)$. Since $y \in T x$ and $H(T x, T g(x)) \leq k\|x-g(x)\|$, there exists $u \in T g(x)$ such that $\|u-y\| \leq$ $\ell\|g(x)-x\|$. Thus

$$
\operatorname{dist}(g(x), T g(x)) \leq \ell\|g(x)-x\|+(1-\mu)\|x-y\|+\varepsilon \mu \operatorname{dist}(x, T x) .
$$

It can be shown that this implies

$$
\begin{aligned}
\operatorname{dist}(g(x), T g(x)) \leq & \ell\|x-g(x)\|+\operatorname{dist}(x, T x) \\
& -(\ell+b)\|x-g(x)\|-(\varepsilon-1) \mu \varepsilon^{\prime}(1-\mu)
\end{aligned}
$$

and thus

$$
b|| x-g(x) \| \leq \operatorname{dist}(x, T x)-\operatorname{dist}(g(x), T g(x))-(\varepsilon-1) \mu \varepsilon^{\prime}(1-\mu) .
$$

At this point it is tempting to say that since $\varepsilon^{\prime}>0$ is arbitrary, it follows that

$$
\|x-g(x)\| \leq \varphi(x)-\varphi(g(x)), \quad x \in D,
$$

where $\varphi(x)=b^{-1} \operatorname{dist}(x, T x)$, whence Caristi's theorem applies. Unfortunately, however, the choice of $y$, and hence $g(x)$, depends on $\varepsilon^{\prime}$. On the other hand, with 
the assumption that $T x$ is proximinal relative to $x$, it is not necessary even to introduce $\varepsilon^{\prime}$. We can merely take $y$ in the above argument to be the point of $T x$ which is nearest $x$. This is, in fact, precisely the observation of $\mathrm{Xu}$ [34] who does assume that the sets $T x$ are all proximinal relative to $x$.

Now we briefly describe how Lim proceeds to obtain the full result using transfinite induction.

Proof of Theorem 4.1 (Outline). Let $\ell \in(k, 1)$ where $k$ is the Lipschitz constant of $T$ and choose $\varepsilon \in(0,1)$ so that

$$
b:=\frac{1-\varepsilon}{1+\varepsilon}-\ell>0
$$

Let $x_{0} \in D$ and choose $y_{0} \in T x_{0}$. We assume $T$ is fixed point free and proceed by transfinite induction. Let $\Omega$ denote the first uncountable ordinal, let $\gamma \in \Omega$, and suppose $y_{\alpha}, x_{\alpha}$ have been defined for all $\alpha<\beta<\gamma$ so that

(i) $y_{\alpha} \in T x_{\alpha}$;

(ii) $x_{\alpha} \neq x_{\alpha+1}$;

(iii) $b \max \left\{\left\|x_{\beta}-x_{\alpha}\right\|,(1 / \ell)\left\|y_{\beta}-y_{\alpha}\right\|\right\} \leq\left\|x_{\alpha}-y_{\alpha}\right\|-\left\|x_{\beta}-y_{\beta}\right\|$.

We proceed to define $y_{\gamma}, x_{\gamma}$ so that (i), (ii), and (iii) remain valid for all $\alpha<$ $\beta \leq \gamma$.

Case 1. Suppose $\gamma=\mu+1$. Since $y_{\mu} \in T x_{\mu}$ and $T$ is fixed point free, we have $\left\|x_{\mu}-y_{\mu}\right\|>0$. By the weak inwardness of $T$, there exist $x_{\mu+1} \in D$ and $\lambda_{\mu+1} \geq 1$ such that

$$
\left\|y_{\mu}-\left(x_{\mu}+\lambda_{\mu+1}\left(x_{\mu+1}-x_{\mu}\right)\right)\right\| \leq \varepsilon\left\|x_{\mu}-y_{\mu}\right\| .
$$

Since $y_{\mu} \in T x_{\mu}$ and

$$
H\left(T x_{\mu+1}, T x_{\mu}\right) \leq k\left\|x_{\mu+1}-x_{\mu}\right\|
$$

there exists $y_{\mu+1} \in T x_{\mu+1}$ such that $\left\|y_{\mu+1}-y_{\mu}\right\| \leq \ell\left\|x_{\mu+1}-x_{\mu}\right\|$. (Note: $y_{\mu+1}$ depends on both $y_{\mu}$ and the contractive condition. It is not the point of $T x_{\mu+1}$ which nearest $x_{\mu+1}$.)

Case 2. Suppose $\gamma$ is a limit ordinal. This case is fairly straightforward by passing to limits.

Once the induction is complete, let $s_{\alpha}=\left\|y_{\alpha}-x_{\alpha}\right\|$. Since $\left\{s_{\alpha}\right\}_{\alpha \in \Omega}$ is decreasing and bounded below, it must be eventually constant. If $\gamma<\Omega$ is such that $s_{\alpha}=s_{\beta}$ for $\alpha, \beta \geq \gamma$, then by (iii), $x_{\alpha+1}=x_{\alpha}$, contradicting (ii). Therefore, $T$ has a fixed point.

While Caristi's theorem seems inadequate for Theorem 4.1, it would be interesting to know if Theorem 4.1 is consequence of the Brézis-Browder order principle (see [2]). (See the discussion in [20], also [1, page 26].) 


\section{An extension of Caristi's theorem}

It is well known that if $T: M \rightarrow M$ is a contraction mapping with Lipschitz constant $k \in(0,1)$, then

$$
d(x, T(x)) \leq \varphi(x)-\varphi(T(x)), \quad x \in M,
$$

where $\varphi: M \rightarrow \mathbb{R}$ is given by $\varphi(x)=(1-k)^{-1} d(x, T(x))$. It is also well known that $T: M \rightarrow M$ has a unique fixed point if for some integer $p>1$, the mapping $T^{p}$ is a contraction mapping. This latter assumption leads to the inequality

$$
d\left(x, T^{p}(x)\right) \leq \psi(x)-\psi\left(T^{p}(x)\right), \quad x \in M,
$$

where $\psi=(1-k)^{-1} d\left(x, T^{p}(x)\right)$. On the other hand, the assumption that $T^{p}$ is a contraction mapping also leads to the inequality

$$
d(x, T(x)) \leq \varphi(x)-\varphi\left(T^{p}(x)\right), \quad x \in M,
$$

with $\varphi$ as above. This raises the obvious question of whether it is possible to replace condition (5.1) with condition (5.3). The answer is "yes" provided that $\left\{\varphi\left(T^{n}(x)\right)\right\}$ is decreasing, but it is not obvious that this fact follows from either Caristi’s theorem or the Brézis-Browder order principle.

Theorem 5.1 ([20]). Let $(M, d)$ be a complete metric space and suppose $T: M \rightarrow$ $M$ is an arbitrary mapping which satisfies

$$
d(x, T(x)) \leq \varphi(x)-\varphi\left(T^{p}(x)\right),
$$

for all $x \in M$, where $p \in N$ is fixed and $\varphi: M \rightarrow R$ is lower semicontinuous and bounded below. Suppose also that $\varphi(T(x)) \leq \varphi(x)$ for each $x \in M$. Then $T$ has a fixed point in $M$.

Proof(Outline). Let

$$
\Phi(x)=\sum_{i=0}^{p-1} \varphi\left(T^{i}(x)\right), \quad x \in M .
$$

Then (5.4) reduces to

$$
d(x, T(x)) \leq \Phi(x)-\Phi(T(x)) .
$$

(Notice that Theorem 4.2 cannot be applied directly to $T$ and $\Phi$ to obtain Theorem 5.1 because the lower semicontinuity assumption on $\varphi$ does not carry over to $\Phi$.) An alternate strategy is to proceed by transfinite induction. Let $x_{0} \in$ $M$, let $\Omega_{1}$ denote the smallest uncountable ordinal, let $\beta \in \Omega_{1}$, and suppose for each $\alpha \in \Omega_{1}$ with $\alpha<\beta, x_{\alpha}$ has been defined so that

(1) $\left\{\varphi\left(x_{\alpha}\right)\right\}_{\alpha<\beta}$ is nonincreasing; 
(2) $\mu \leq \alpha \Rightarrow d\left(x_{\mu}, x_{\alpha}\right) \leq \Phi\left(x_{\mu}\right)-\Phi\left(x_{\alpha}\right)$;

(3) $\mu<\alpha \Rightarrow x_{\mu+1}=T\left(x_{\mu}\right)$.

It is possible to define $x_{\beta}$ and show that (1), (2), and (3) hold for $\alpha \leq \beta$. The subtlety is in showing that (2) holds when $\beta$ is a limit ordinal. However, once this is done, $\left\{x_{\alpha}\right\}$ is defined for all $\alpha \in \Omega_{1}$. Since $\left\{\Phi\left(x_{\alpha}\right)\right\}$ is nonincreasing on $\left\{x_{\alpha}\right\}$ and $\Omega_{1}$ is uncountable, there must exist $\alpha_{0} \in \Omega_{1}$ such that $\Phi\left(x_{\alpha}\right)$ is constant for all $\alpha \geq \alpha_{0}$. This clearly implies that $T\left(x_{\alpha_{0}}\right)=x_{\alpha_{0}}$.

5.1. Remarks. One of the main objectives of [20] was to show, by using the Brézis-Browder order principle, that the lower semicontinuity condition in Theorem 4.2 can be weakened so that the resulting theorem contains the extension of Caristi's theorem given in [10]. In particular, it suffices to assume that $\varphi: M \rightarrow \mathbb{R}$ is lower semicontinuous from above. This means that given any sequence $\left\{x_{n}\right\}$ in $M$, the conditions $\lim _{n} x_{n}=x$ and $\varphi\left(x_{n}\right) \downarrow r \Rightarrow \varphi(x) \leq r$. In fact, an inspection of the proof of [20, Theorem 2.1] shows that an even weaker assumption suffices; namely, it is enough to assume that $\lim _{n} x_{n}=x$ and $x_{n} \preceq$ $x_{n+1} \Rightarrow \varphi(x) \leq r$, where $\preceq$ is defined by: $x \preceq y \Longleftrightarrow d(x, y) \leq \varphi(x)-\varphi(y)$. The same reasoning applies to Theorem 5.1. (Actually, this general idea seems to have arisen earlier in [11], where Gajek and Zagrodny use the notion of lower semicontinuous from above, which they call decreasingly lower semicontinuous to establish an extension of Ekeland's principle.)

We might also wonder whether it is possible to allow $p$ in Theorem 5.1 to depend on $x$. However, this weaker assumption does not even imply that the orbits of $T$ are bounded.

\section{Historical comments}

In some sense, the very origins of metric fixed-point theory are rooted in transfinite induction. Indeed, Brodskiľ and Mil'man, in their seminal paper [3], used transfinite induction to show that if a subset $K$ of a Banach space has "normal structure and is compact in some topology $\tau$ for which the normed closed balls are $\tau$-closed (e.g., the weak or weak* topology), then $K$ contains a uniquely determined point (called the center of $K$ ) which is fixed under every isometry of $K$ onto itself. Other early uses of transfinite induction include the work of Sadovskii on condensing operators ([30,31]; although in the latter instance, an elementary proof, without transfinite induction, has been given by Reich [27]; (see also [25]). Altman [1] makes heavy use of transfinite methods in his study of contractors and contractor directions. Here again, however, other methods sometimes suffice (e.g., see [7, 10]). In [17], the theory of ultranets is used to define a transfinite iteration process. As a consequence, it is noted that given any weakly compact set $K$ and any contractive mapping $T$ of $K$ into $K$ (i.e., $\|T(x)-T(y)\|<\|x-y\|$ for $x, y \in K, x \neq y)$, there is a unique point $z \in K$ such that $T^{\Omega}(x)=z$ for each $x \in K$. In [26], conditions, under which this point $z$ is 
actually a fixed point of $T$, are discussed. Finally, we also mention that Wong subsequently gave a simpler transfinite induction proof of Caristi's theorem in [32].

\section{Acknowledgment}

The author thanks the referee for giving attention to some typographical oversights in the original draft of this manuscript and for suggesting several helpful citations.

\section{References}

[1] M. Altman, Contractors and Contractor Directions. Theory and Applications, Lecture Notes in Pure and Applied Mathematics, vol. 32, Marcel Dekker, New York, 1977.

[2] H. Brézis and F. E. Browder, A general principle on ordered sets in nonlinear functional analysis, Advances in Math. 21 (1976), no. 3, 355-364.

[3] M. S. Brodskil and D. P. Mil'man, On the center of a convex set, Dokl. Akad. Nauk SSSR (N.S.) 59 (1948), 837-840 (Russian).

[4] G. Cantor, Beiträge zur begründung der transfiniten Mengenlehre. Part II, Math. Ann. 49 (1897), 207-246 (German).

[5] J. Caristi, Fixed point theorems for mappings satisfying inwardness conditions, Trans. Amer. Math. Soc. 215 (1976), 241-251.

[6] F. H. Clarke, Pointwise contraction criteria for the existence of fixed points, Canad. Math. Bull. 21 (1978), no. 1, 7-11.

[7] W. J. Cramer Jr. and W. O. Ray, Solvability of nonlinear operator equations, Pacific J. Math. 95 (1981), no. 1, 37-50.

[8] J. W. Dauben, Georg Cantor. His Mathematics and Philosophy of the Infinite, Harvard University Press, Massachusetts, 1979.

[9] K. Deimling, Multivalued Differential Equations, de Gruyter Series in Nonlinear Analysis and Applications, vol. 1, Walter de Gruyter, Berlin, 1992.

[10] D. Downing and W. A. Kirk, A generalization of Caristi's theorem with applications to nonlinear mapping theory, Pacific J. Math. 69 (1977), no. 2, 339-346.

[11] L. Gajek and D. Zagrodny, Geometric variational principle. Different aspects of differentiability (Warsaw, 1993), Dissertationes Math. (Rozprawy Mat.) 340 (1995), $55-71$.

[12] K. Goebel, On a fixed point theorem for multivalued nonexpansive mappings, Ann. Univ. Mariae Curie-Skłodowska Sect. A 29 (1975), 69-72 (1977).

[13] S. Ishikawa, Fixed points and iteration of a nonexpansive mapping in a Banach space, Proc. Amer. Math. Soc. 59 (1976), no. 1, 65-71.

[14] M. A. Khamsi, On metric spaces with uniform normal structure, Proc. Amer. Math. Soc. 106 (1989), no. 3, 723-726.

[15] W. A. Kirk, An application of a generalized Krasnosel'skiu-Ishikawa iteration process, Fixed Point Theory and Applications (Marseille, 1989), Pitman Res. Notes Math. Ser., vol. 252, Longman Sci. Tech., Harlow, 1991, pp. 261-268.

[16] Compactness and countable compactness in weak topologies, Studia Math. 112 (1995), no. 3, 243-250. 
[17] W. A. Kirk and S. Massa, An ultranet technique in metric fixed point theory, Topics in Mathematical Analysis, Ser. Pure Math., vol. 11, World Sci. Publishing, New Jersey, 1989, pp. 539-546.

[18] W. A. Kirk and W. O. Ray, A note on Lipschitzian mappings in convex metric spaces, Canad. Math. Bull. 20 (1977), no. 4, 463-466.

[19] - A remark on directional contractions, Proc. Amer. Math. Soc. 66 (1977), no. 2, 279-283.

[20] W. A. Kirk and L. M. Saliga, The Brézis-Browder order principle and extensions of Caristi's theorem, Nonlinear Anal. 47 (2001), no. Ser. A: Theory Methods, 27652778 .

[21] J. Kulesza and T.-C. Lim, On weak compactness and countable weak compactness in fixed point theory, Proc. Amer. Math. Soc. 124 (1996), no. 11, 3345-3349.

[22] T.-C. Lim, A fixed point theorem for multivalued nonexpansive mappings in a uniformly convex Banach space, Bull. Amer. Math. Soc. 80 (1974), 1123-1126.

[23] - A fixed point theorem for weakly inward multivalued contractions, J. Math. Anal. Appl. 247 (2000), no. 1, 323-327.

[24] C. Martínez-Yañez, A remark on weakly inward contractions, Nonlinear Anal. 16 (1991), no. 10, 847-848.

[25] P. Massatt, Some properties of condensing maps, Ann. Mat. Pura Appl. (4) 125 (1980), 101-115.

[26] A. A. Melentsov, Iterative nets of weakly contractive operators, Acta Comment. Univ. Tartu. Math. (1996), no. 1, 9-12 (Russian).

[27] S. Reich, Fixed points of condensing functions, J. Math. Anal. Appl. 41 (1973), 460467.

[28] _ On fixed point theorems obtained from existence theorems for differential equations, J. Math. Anal. Appl. 54 (1976), no. 1, 26-36.

[29] Approximate selections, best approximations, fixed points, and invariant sets, J. Math. Anal. Appl. 62 (1978), no. 1, 104-113.

[30] B. N. Sadovskiǔ, On a fixed point principle, Funkcional. Anal. i Priložen. 1 (1967), no. 2, 74-76 (Russian).

[31] Limit-compact and condensing operators, Uspehi Mat. Nauk 27 (1972), no. 1(163), 81-146 (Russian), English translation: Russian Math. Surveys 27 (1972), 85-155.

[32] C.-S. Wong, On a fixed point theorem of contractive type, Proc. Amer. Math. Soc. 57 (1976), no. 2, 283-284.

[33] H.-K. Xu, Metric fixed point theory for multivalued mappings, Dissertationes Math. (Rozprawy Mat.) 389 (2000), 39.

[34] - Multivalued nonexpansive mappings in Banach spaces, Nonlinear Anal. 43 (2001), no. 6, Ser. A: Theory Methods, 693-706.

[35] H. W. Yi and Y. C. Zhao, Fixed point theorems for weakly inward multivalued mappings and their randomizations, J. Math. Anal. Appl. 183 (1994), no. 3, 613-619.

W. A. Kirk: Department of Mathematics, The University of Iowa, Iowa City, IA 522421419, USA

E-mail address: kirk@math.uiowa.edu 


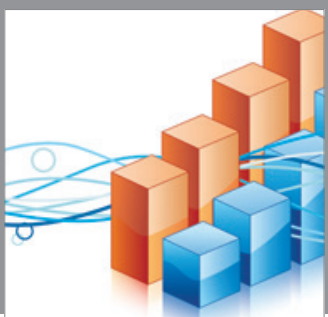

Advances in

Operations Research

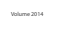

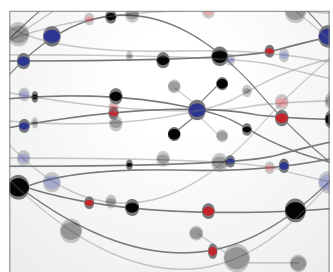

\section{The Scientific} World Journal
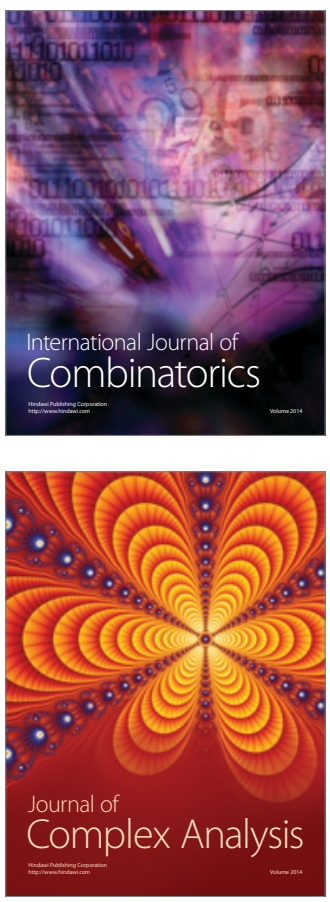

International Journal of

Mathematics and

Mathematical

Sciences
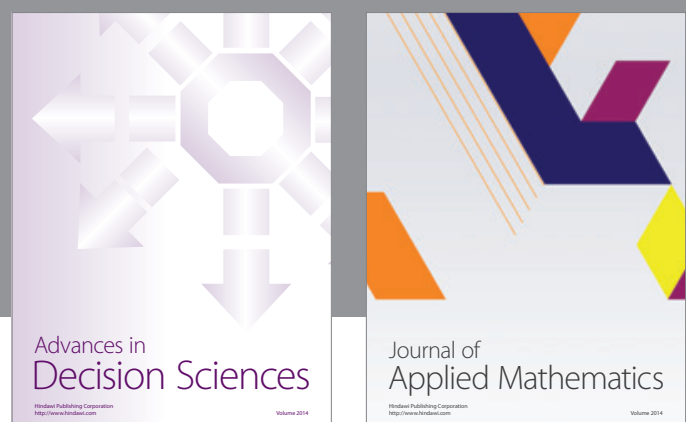

Journal of

Applied Mathematics
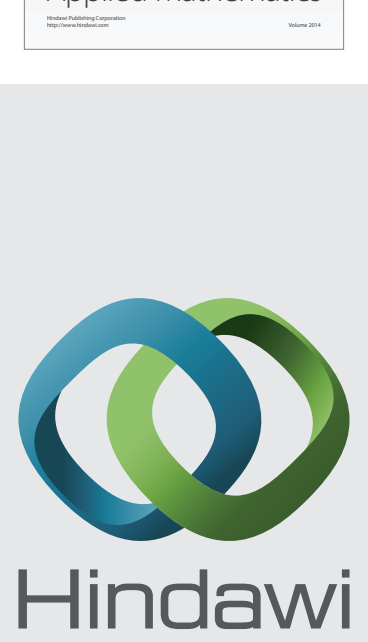

Submit your manuscripts at http://www.hindawi.com
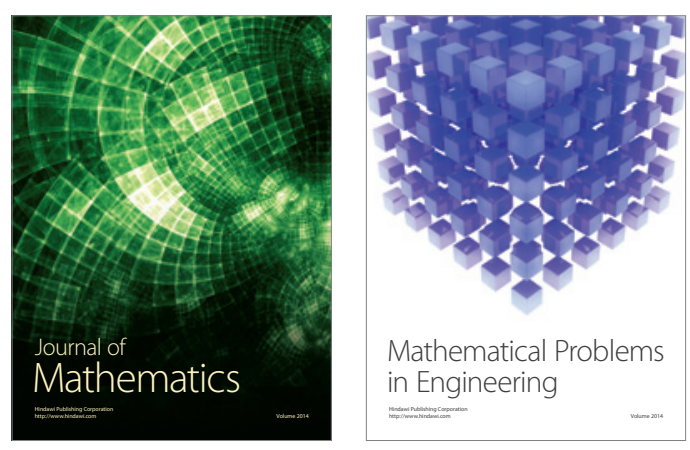

Mathematical Problems in Engineering
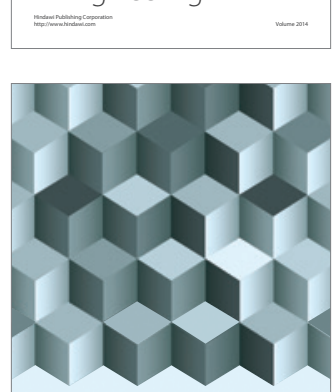

Journal of

Function Spaces
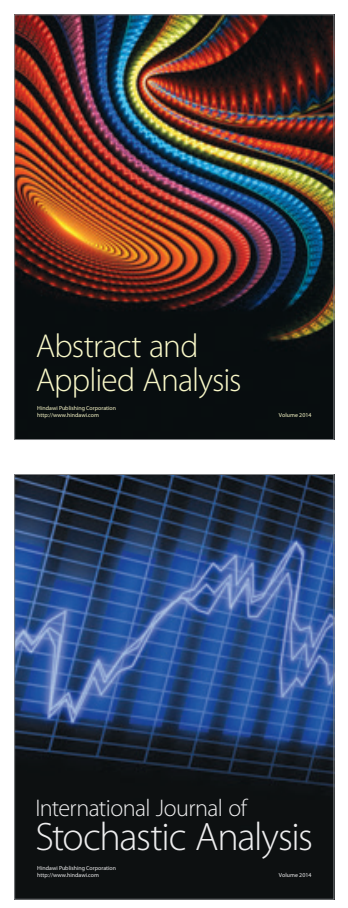

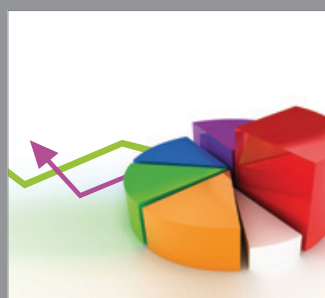

ournal of

Probability and Statistics

Promensencen
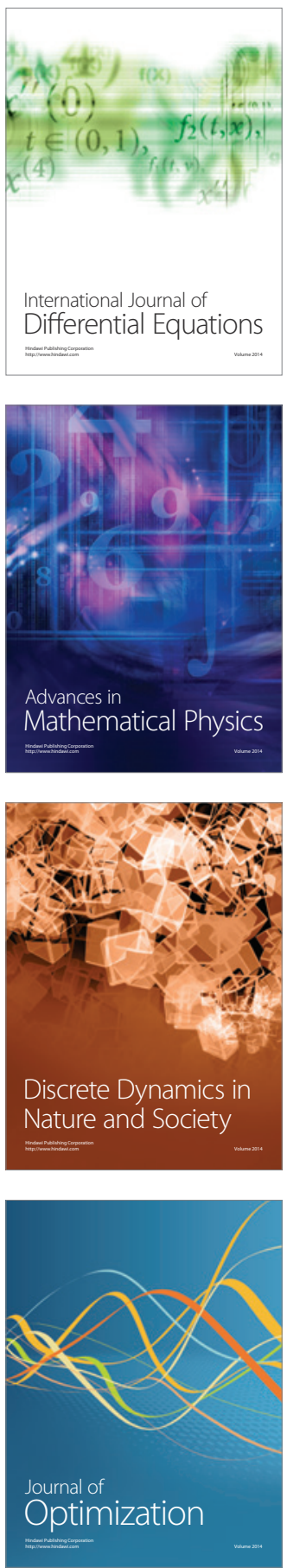